\title{
Characteristic Analysis of a Difference-Based Cascade Thermocouple Measurement System in Steady Flow
}

\author{
Zhaoxin Yang \\ China Aerodynamics Research and Development \\ Center \\ Mianyang, Sichuan \\ e-mail: tutu198210@sina.com \\ Wen Gai \\ China Aerodynamics Research and Development \\ Center \\ Mianyang, Sichuan \\ e-mail: 2420427001@qq.com
}

\author{
Jie Han \\ China Aerodynamics Research and Development \\ Center \\ Mianyang, Sichuan \\ e-mail: jhan-ln@yahoo.com.cn
}

\begin{abstract}
The dynamic characteristics evaluation of thermocouples presents a challenging problem for temperature measurement. The authors have recently proposed a novel difference-based cascade thermocouple measurement system that consists of three thermocouples with different time constants. The combination of the test system with use of the Tikhonov algorithm for parameter identification provides excellent time constant estimation despite the lack of any a priori assumption on the time constant ratios of the thermocouples. The developed scheme has been tested in a generator of a step excitation temperature signal device with constant flow and the simulated results show that the proposed scheme is effective.
\end{abstract}

Keywords- difference-based Cascade; thermocouple; time Constant Estimation; identification; mathmatical model

\section{INTRODUCTION}

The advantage of thermocouple is distinctive in engineering applications with respect to its low cost and robustness [1]. However, thermocouple is contact-type sensor with a sensing process based on heat balance. Heat inertia causes severe errors in response to transient temperature $[2,3]$. Therefore, a compromise should be made between dynamic measurement and robustness [4, 5]. When the surrounding condition is invariant during sensor operation, a thermocouple is generally considered as a first-order linear dynamic model [6], and its dynamic characteristics are represented by the time constant. Therefore, acquisition of a reliable measurement of transient temperature depends on the accurate estimation of the time constant of thermocouples intensively.

Generally, the dynamic model is established by aid of the excitation and response signals of the test system [7]. The model parameters estimation is obtained by means of system identification which is relative maturity in classical algorithms. Then the dynamic parameters which characterize the dynamic performance of the system can be obtained from the mathematical model. Furthermore, the dynamic performance of the testing system can be improved based on the dynamic parameters [8].

This paper proposed a novel method for calculating the dynamic parameters of the first-order testing system. On the basis of the characteristics of a first-order dynamic system, the dynamic performance parameters of the system can be estimated by the difference-based cascade between thermocouples (DBCT). The DBCT test system is composed of three thermocouples with different diameters which have to be mounted as close as possible to test the same temperature at the same point. Therefore the excitation signals of the thermocouples are considered equal. The difference between the thermocouple responses is obtained to establish the mathematical model in this study. The time constants of each thermocouple of the DBCT test system can be solved by identifying the parameters of the mathematical model.

The method is characterized in that: without the tests for the thermocouple excitation signal which requires trace to the source in dynamic measurement, time constants of DBCT test system are identified and estimated by means of system identification method based on the established new mathematical model. If the position deviation is disregarded in installation procedure, the influence of environmental noise or other factors on each thermocouple at the same test point becomes consistent. The information matrix of the system composed of the sequences of the temperature response difference of each thermocouple decreases the influence of environmental factors on the accuracy of time constant identification. Generalized total least square algorithm based on Tikhonov regularization theory (GTLS-Tikhonov) is used to estimate the parameters of the DBCT test system model. For the noise between the columns of the augmented information matrix is no longer uncorrelated, (GTLS) has to be used to 
provide consistent and unbiased estimates of the parameters [9]. The time constants are then calculated, and the influence of noise on the estimated results is analyzed. In order to overcome the information matrix collinearity caused by the physical characteristics of the time constants of the thermocouple, Tikhonov regularization method based on singular value decomposition (Tikhonov-SVD) is employed to optimize the weighted advance identification. In this way, the implementation conditions of the DBCT test method are extended. Finally, this approach is verified through practical engineering application. The processing ability of Tikhonov-SVD is tested in terms of noise reduction during time constant estimation and solving the collinearity of DBCT test system.

\section{TEST SYSTEM}

Three thermocouples of the DBCT test system with different time constants which considered as the firstorder low-pass filter can be expressed as [10]:

$$
H_{1}=\frac{1}{1+\tau_{1} s}, H_{2}=\frac{1}{1+\tau_{2} s} H_{3}=\frac{1}{1+\tau_{3} s}
$$

where $H$ is the thermocouple transfer function. The subscripts 1, 2 and 3 mark the parameters and signals of the first, second and third channel of the test system, respectively.

The parallel connection system is shown in Fig. 1.

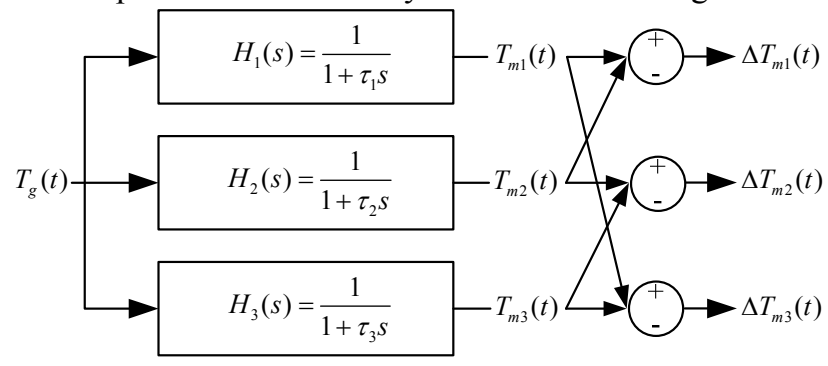

Figure. 1. Diagram of the time constant estimation of DBCT test system

As shown in Fig. 1, the responses of three thermocouples to the same excitation signal $T_{g}(t)$ are $T_{m 1}(t), T_{m 2}(t), T_{m 3}(t)$ for three different channels of the system, and the corresponding differences signals are $\Delta$ $T_{m 1}(t), \Delta T_{m 2}(t), \quad \Delta T_{m 3}(t)$, respectively.

where

$$
\begin{aligned}
\Delta T_{m 1}(k) & =T_{m 2}(k)-T_{m 1}(k) \\
\Delta T_{m 2}(k) & =T_{m 3}(k)-T_{m 2}(k) \\
\Delta T_{m 3}(k) & =T_{m 3}(k)-T_{m 1}(k)
\end{aligned}
$$

The discretization of (2) get by zero-order holder with the assumed sampling interval $\lambda_{0}$ transformed into matrix form as follows:

$$
Y=A X
$$

where

$$
\begin{aligned}
Y & =\left[\Delta T_{m 3}(2), \Delta T_{m 3}(3), \cdots, \Delta T_{m 3}(L)\right]^{T} \\
A & =\left[\begin{array}{ccc}
\Delta T_{m 3}(1) & \Delta T_{m 2}(2) & \Delta T_{m 3}(1) \\
\Delta T_{m 3}(2) & \Delta T_{m 2}(3) & \Delta T_{m 3}(2) \\
\vdots & \vdots & \vdots \\
\Delta T_{m 3}(L-1) & \Delta T_{m 2}(L) & \Delta T_{m 3}(L-1)
\end{array}\right]
\end{aligned}
$$

$$
\begin{gathered}
X=\left[\begin{array}{lll}
\beta_{1} & \beta_{2} & \beta_{3}
\end{array}\right], \text { and } \\
\beta_{1}=\alpha_{3}, \beta_{2}=\frac{\alpha_{1}-\alpha_{3}}{\alpha_{1}-\alpha_{2}}, \beta=-\alpha_{2} \cdot \beta_{2}
\end{gathered}
$$

The system described in (3) is called $\beta$ system, which is a linear system. Bases on the $\beta$ system, the DBCT test system which use difference signal as the excitation and response signal is believed to be more moderate to background measurement noise and numerical errors than those used single signal as test signal. The reason for this is that the use of difference signal, which minimizes the effect of measurement signal errors, enables more stable $X$ optimization.

\section{IDENTIFICATION METHOD}

For the $\beta$ system, the information matrix $A$ is obtained by calculating the differences among the responses of the three thermocouples, in which the test error of the system is included. The differences of the thermocouples constitute the $\beta$ system excitation and response signal. So the errors in each column of the augment matrix [A Y] are no longer uncorrelated. Generalized total least squares (GTLS) method should be used to compute consistent estimates of parameters. Compared with ordinary least squares algorithm (LS) method which considered the error only exists in the measurement matrix $Y$ and the information matrix $A$ does not contain any error, and the total least square(TLS) method, which consider A and Y are uncorrelated with zero mean and equal variance, such method is more efficient. Thus, a more reliable model is established to reasonably allocate the error into the information and observation matrices, with the constraint conditions expressed as follows:

$$
\min \left\|(A, Y)-\left(A^{\#}, Y^{\#}\right)\right\|_{F},
$$

where $A^{\#} X=Y^{\#}, A^{\#}$, and $Y^{\#}$ are the information and observation matrices without the system test error, respectively.

The ill-posed problems of GTLS can be solved by regularization reduction based on the theory of numerical analysis. In this way, a greater condition number can be achieved. However, more ill-posed or unstable results may appear in the solution. According to the theory of mathematical statistics, one coefficient of the approximate covariance matrix $\left(\sigma_{n+1} I\right)$ of the error is subtracted from the solution of GTLS method to remove the deviation of the multitrait-multimethod matrix $A^{T} A$ to a certain degree. Consequently, a relatively stable solution is obtained.

\section{EFFECT OF Noise LeVEls ON THE TIME CONSTANT IDENTIFICATION OF THE DBCT TEST SYSTEM}

The algorithm variation in identification error of time constants with different noise levels is compared with the GTLS-Tikhonov algorithm estimation error of time constants with the sinusoidal and step excitation signals, as shown in Figs. 2 and 3. 


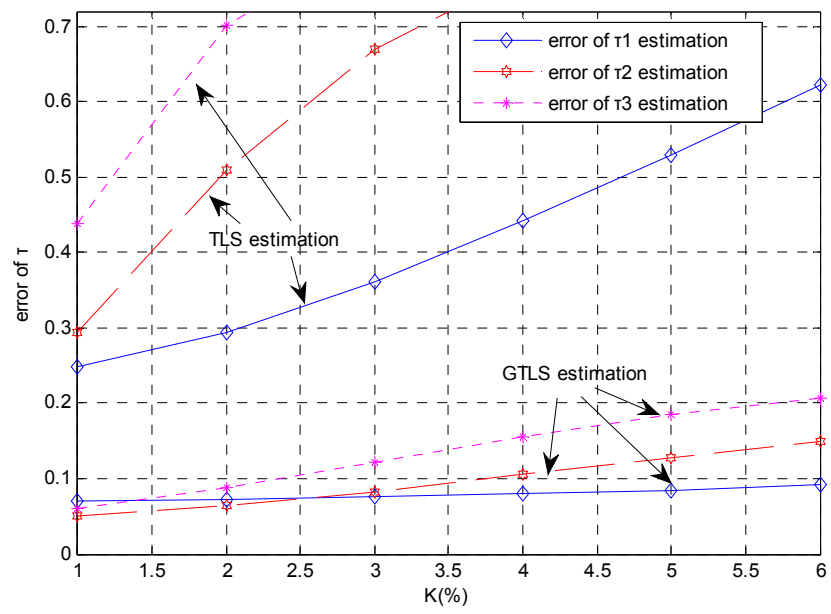

Figure. 2 Relationship between $\mathrm{K}$ and $\mathrm{e}_{\tau}$ with sinusoidal excitation signal

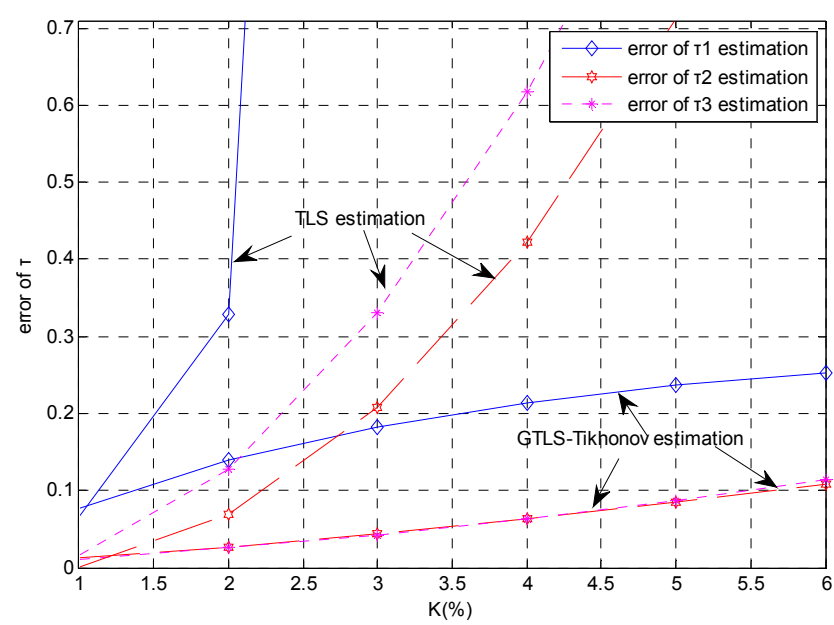

Figure. 3 Relationship between $\mathrm{K}$ and $\mathrm{e}_{\tau}$ with an ideal step excitation signal

Figs. 2 and 3 show that in terms of time constant estimation error, when $\delta<\left\|X_{L S}\right\|_{2}$, the accuracy of the GTLS-Tikhonov and TLS algorithms are comparable at the noise level of $K=0$. However, the results of the TLS algorithm rapidly diverge with an increase in noise level. When the noise level reaches a certain degree, the TLS algorithm would not yield reasonable identification results. Except at $K=0$, the accuracy of time constant identification by the GTLS-Tikhonov algorithm is relatively high compared with that by the TLS algorithm. However, the error by GTLS-Tikhonov algorithm also monotonically increases with the $K$ value. A certain noise level must be satisfied to enable the GTLS-Tikhonov algorithm to yield a reasonable identification result.

\section{EXPERIMENT APPLICATION}

To test the algorithms performance for time constant identification of DBCT test system, experiments were carried out to acquire the responses of thermocouples in an air stream with a constant velocity, based on a step temperature signal generator device which specifically designed to produce step temperature fluctuations at constant fluid.

The step temperature signal generator device consists of five parts: air storage chamber, circulating device, thermal power device, membranes rupture device, and thermal insulation device. DBCT test system is comprised of three $K$-type thermocouples which placed in the same sensor shell to minimize the offset errors caused by the installation error among the thermocouples. The thermocouple wire installed downstream of the air storage chamber with the diameters of $d_{1}=0.2 \mathrm{~mm}, d_{2}=0.3 \mathrm{~mm}$, and $d_{3}=0.8 \mathrm{~mm}$ corresponding to the three channels of DBCT system. The throttle nozzle is installed at the exit of the chamber to provide the constant fluid velocity in the chamber. The air storage chamber is a closed chamber wrapped in thermal insulation material. The schematic diagram is shown in Fig. 4 to illustrate the process of the time constant estimation of the DBCT test system.

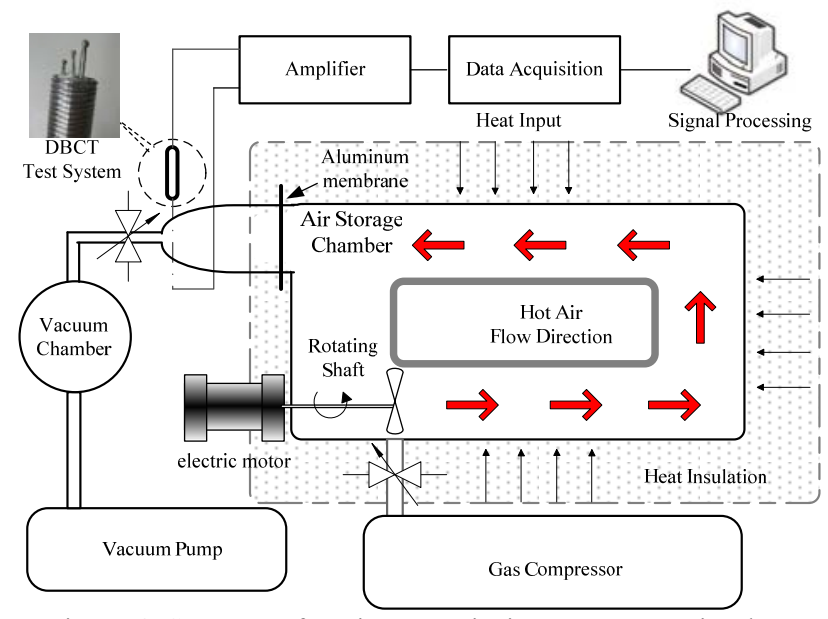

Figure. 4. Generator of an air step excitation temperature signal

The results of time constant estimation by frequency domain modeling, TLS algorithm, GTLS- Tikhonov, and SVD-Tikhonov algorithm are shown in TABLE I .

TABLE I. Estimates of time constants based on measured data

\begin{tabular}{|c|c|c|c|c|}
\hline \multirow{2}{*}{$\begin{array}{c}\text { Time } \\
\text { Constant }\end{array}$} & \multirow{2}{*}{$\begin{array}{c}\text { Frequency } \\
\text { domain modeling }\end{array}$} & \multicolumn{3}{|c|}{ The algorithm estimates (s) } \\
\cline { 3 - 5 } & LS & GR-TLS & $\begin{array}{c}\text { SVD- } \\
\text { Tikhonov }\end{array}$ \\
\hline$\tau 1(\mathrm{~s})$ & 0.85 & NAN & NAN & 0.58 \\
\hline$\tau 2(\mathrm{~s})$ & 1.673 & NAN & 1.174 & 1.515 \\
\hline$\tau 3(\mathrm{~s})$ & 3.3 & 1.895 & 2.589 & 2.894 \\
\hline
\end{tabular}

So we can get that the error of response of the thermocouples and the identification method selected for the ill-posed problems have significant impact on identification results of DBCT test system. The diameters of the thermocouple wire, $d_{1}$ and $d_{2}$, are very close in the experiment; thus, the returned values, $\tau 1$ and $\tau 2$, of time constants are non-reasonable values because of the collinearity of the information matrix. These results confirm the constraints on the consistency of the physical conditions of the thermocouple wires. SVD-Tikhonov regularization method is used to estimate the time constants, and the estimate results are more reasonable. 


\section{SUMMARY}

This paper presents a new method for estimating the time constants of thermocouples. A mathematical model is established on the basis of the DBCT test system. The difference in thermocouple response is applied to construct the information matrix and the observation sequence. Model parameter estimation is performed after parameter identification. GTLS-Tikhonov algorithm is used to process the errors correlation in the augmented information matrix. In contrast to the LS algorithm and TLS algorithm, the GTLS-Tikhonov algorithm achieves consistent and unbiased parameter estimation. The effects of noise levels on the accuracy of time constant estimation and the effects of time constants differentiation on identification precision are examined. By using the Tikhonov-SVD algorithm, reasonable identification results can be returned even when an ill-posed matrix causes a divergence of the estimation results. In a device that produces a step temperature signal, DBCT system of three thermocouples in the same shell are stimulated. Tikhonov-SVD algorithm is used to estimate the time constants. The time constant identification results verify the effectiveness of the proposed method.

\section{REFERENCES}

[1] K.Danisman, I.Dalkiran, and F.V. Celebi, "Design of a high precision temperature measurement system based on artificial neural network for different thermocouple types", Measurement. vol. 39, pp. 695-700, 2006.

[2] B. Saggin, S. Debei, and M.Zaccariotto. "Dynamic error correction of a thermometer for atmospheric measurement", Measurement. vol. 30 , pp. 223-230, 2011
[3] Helcio R. B. Orlande, George S. Dulikravich, and Markus Neumayer et al. "Accelerated bayesian inference for the estimation of spatially varying heat flux in a heat conduction problem", Numerical Heat Transfer Part A-Applications. vol. 65, pp. 1-25, 2014

[4] Xing Fu, Xiaobing Luo. "Can thermocouple measure surface temperature of light emitting diode module accurately?" International Journal of Heat and Mass Transfer. vol. 65, pp. 199202, 2013.

[5] Shi You-An, Zeng lei, and Qian wei-Qi et al. "A data processing method in the experiment of heat flux testing using inverse methods", Aerospace Science and Technology. vol. 29, pp. 74-80, 2013.

[6] Blaine W. Asay, Steven F. Son, and Peter M. Dickson et al. "An investigation of the dynamic response of thermocouples in inert and reacting condensed phase energetic materials", Propellants Explosives Pyrotechnics. vol. 30, pp. 199-208, 2009

[7] J. Panda, K. B. M. Q. Zaman, and R. G. Seasholtz. "Measurement of initial conditions at nozzle exit of high speed jets", AIAA -20012143, 2014

[8] Abdul Md Mazid, A B M Shawkat Ali. "Opto-tactile Sensor for Surface Texture Pattern Identification using Support Vector Machine", 10th Intl. Conf. on Control, Automation, Robotics and Vision. Hanoi, Vietnam, 2008, pp. 1830-1835.

[9] Mejdell T, Skogestad S. "Output estimation using multiple secondary measurement: high-purity distillation", AIChE.J, vol. 39, pp. 1641-1653, 2013.

[10] Jamaluddin, Hishamuddin, Abd. Samad, M. F. et al. "Optimum grouping in a modified genetic algorithm for discrete-time, nonlinear system identification". Journal of Systems and Control Engineering, vol. 221, pp. 975-989, 2007. 